Military Technical College

Kobry El-Kobba

Cairo, Egypt

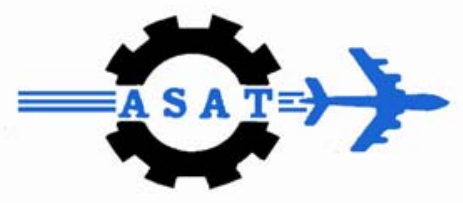

12-th International Conference

on

Aerospace Sciences \&

Aviation Technology

\title{
LAPLACIAN FILTERED MINIMUM SHIFT KEYING
}

\author{
IBRAHIM* N. ABU-ISBEIH
}

\section{ABSTRACT}

This paper describes a new method of premodulation filtered MSK called Laplacianfiltered minimum shift keying (LMSK). LMSK is proposed as a method for achieving good spectral efficiency over regular MSK and it turns out to achieve improvement in error rate performance over the known Gaussian filtered minimum shift keying (GMSK). To compare the behavior of the system under the proposed new technique, bandwidth efficiency (i.e., bps/Hz) and fractional out-of-band power is used. As well, error rate performance in such systems is used in the evaluation of this work.

\section{KEY WORDS}

Minimum Shift Keying (MSK), Laplacian MSK.

\section{NOMENCLATURE}

FM: Frequency Modulation.

GMSK: Gaussian Minimum Shift Keying.

LMSK: Laplacian Minimum Shift Keying.

MSK: Minimum Shift Keying.

NRZ: Non Return to Zero.

SCPC: Single-Channel-Per-Carrier

VCO: Voltage Controlled Oscillator. 


\section{INTRODUCTION}

Digital-frequency and phase-modulation techniques have been increasingly important in radio, microwave, and optical communication systems [1]. For radio communication, constant-envelope modulated signals are preferable due to existing system constraints in power economy and the consequent use of non-linear power amplifiers. Quite naturally this leads to the use of frequency modulation as one possibility. However, the spectrum of an FM signal is relatively wide. In order to narrow the spectrum, a channel filter with a precisely prescribed attenuation and phase characteristics may be used; but this is not attractive in radio equipment. Besides, a filter of this kind cannot be used if the transmitted center frequency has to be changed.

Another method for narrowing the spectrum is to shape the data at the input of the frequency modulator by means of a filter [2]. GMSK (Gaussian-filtered minimum shift keying) $[3,4]$, in which a premodulation Gaussian filter is introduced to MSK, and is one of the promising modulation schemes for single-channel-per-carrier (SCPC) digital mobile communications in terms of its narrow-band and sharp cut-off property of the power spectrum and its constant envelope property [4]. While the premodulation filtering is very effective in reducing the out-of-band power of the modulated wave, it causes degradation of bit-error-rate (BER) performance versus signal-to-noise ratio due to the increased intersymbol interference $[3,4,5]$. In this work, premodulation Laplacian filtered minimum shift keying (LMSK) is proposed as an effective digital modulation, and is compared to GMSK.

\section{SPECTRUM MANIPULATION OF LMSK}

The LMSK modulator can be realized as shown in Fig.I, where a premodulation baseband Laplacian low-pass filter is added to an MSK modulator which is a continuous-phase digital FM with modulation index of 0.5 . If the area of the filtered output pulse corresponding to each bit is combined to the phase shift of $\pm \pi / 2$, a carrier wave can be recovered from the modulated wave. Thus, coherent detection is possible for the LMSK modulated wave.

Let the non-return-to-zero (NRZ) input signal to the baseband low-pass filter be

$m(t)=\sum_{k=-\infty}^{\infty} a_{k} \cdot b(t-k T)$

where $\mathrm{T}$ is the bit duration, $a_{k}$ is the random equiprobable data assuming the values +1 or -1 and

$b(t)= \begin{cases}1 & ; 0 \leq t \leq T \\ 0 & ; \text { elsewhere }\end{cases}$ 
The impulse response of the baseband Laplacian filter is given by

$h(t)=\frac{\alpha}{2} e^{-\alpha|t|}, \quad|t|<\infty$

where $\alpha$ is a parameter related to the $3-d B$ filter bandwidth. Consequently, the transfer function of the Laplacian LPF is given by

$$
H(f)=\frac{\alpha^{2}}{\alpha^{2}+(2 \pi f)^{2}}
$$

where the 3-dB filter bandwidth $B_{b}$ can be found to be

$$
B_{b}=\frac{(\sqrt{2}-1)^{1 / 2}}{2 \pi} \cdot \alpha
$$

The Laplacian filtered MSK (LMSK) modulated signal is given by

$$
x(t)=\cos \left[2 \pi f_{c} t+\Phi(t)\right]
$$

where $f_{c}$ is the carrier frequency and $\Phi(t)$ is the phase of the modulated signal.

The Laplacian filtered input to the voltage controlled oscillator (VCO), in Fig.1, can be calculated as

$$
l(t)=\int_{-\infty}^{\infty} h(\tau) m(t-\tau) d \tau
$$

Then, the phase of the LMSK modulated signal is given by

$$
\Phi(t)=\frac{\pi}{2 T} \int_{t_{0}}^{t} l(\xi) d \xi+\Phi\left(t_{0}\right)
$$

where $\Phi\left(t_{o}\right)$ is an initial phase at an arbitrarily selected time $t_{o}$.

By substituting $(I)$ in (7), we then get

$$
\begin{aligned}
l(t) & =\int_{-\infty}^{\infty} h(\tau) \sum_{k=-\infty}^{\infty} a_{k} \cdot b(t-k T-\tau) d \tau \\
& =\sum_{k=-\infty}^{\infty} a_{k} \cdot \int_{-\infty}^{\infty} h(\tau) b(t-k T-\tau) d \tau
\end{aligned}
$$


where

$$
=\sum_{k=-\infty}^{\infty} a_{k} \cdot z(t-k T)
$$

$$
z(t)=\int_{-\infty}^{\infty} h(\tau) b(t-\tau) d \tau
$$

Then, after substituting (11) in (8) we get the phase of $x(t)$ as

$$
\begin{aligned}
\Phi(t) & =\frac{\pi}{2 T} \int_{t_{0}}^{t} \sum_{k=-\infty}^{\infty} a_{k} \cdot z(\xi-k T)+\Phi\left(t_{0}\right) \\
& =\sum_{k=-\infty}^{\infty} a_{k} \cdot g(t-k T)+\Phi\left(t_{0}\right)
\end{aligned}
$$

where

$$
g(t)=\frac{\pi}{2 T} \int_{t_{0}}^{t} z(u) d u
$$

From (2), (3) and (12) we can get $z(t)$ as

$$
Z(t)= \begin{cases}\frac{e^{\alpha t}}{2}\left[1-e^{-\alpha T}\right] & ; t<0 \\ \frac{1}{2}\left[2-e^{-\alpha t}-e^{\alpha(t-T)}\right] & ; 0 \leq t \leq T \\ \frac{e^{-\alpha t}}{2}\left[e^{\alpha T}-1\right] & ; t>T\end{cases}
$$

and from (15) and (16) assuming $t_{o}=-\infty$, we then get

$$
g(t)= \begin{cases}\frac{\pi e^{\alpha t}}{4 \alpha T}\left[1-e^{-\alpha T}\right] & ; t<0 \\ \frac{\pi}{2 T}\left[t+\frac{e^{-\alpha t}}{2 \alpha}-\frac{e^{\alpha(t-T)}}{2 \alpha}\right] & ; 0 \leq t \leq T \\ \frac{\pi e^{-\alpha t}}{4 \alpha T}\left[1-e^{\alpha T}\right]+\frac{\pi}{2} & ; t>T\end{cases}
$$


The functions $z(t)$ and $g(t)$ are shown in Fig.2 and Fig.3, respectively.

Fig.4 shows the output spectrum of the LMSK signal versus the normalized frequency difference from the carrier center frequency $\left(f-f_{c}\right) T$, where the normalized 3-dB bandwidth of the premodulation Laplacian LPF $B_{b} T$ is a parameter of significance. Fig.5 shows the fractional out-of-band power curves versus the normalized one-sided bandwidth WT. Table 1 results the occupied RF bandwidth for the percentage of power where $B_{b} T$ is also a variable parameter. For comparison the occupied bandwidth of Gaussian filtered MSK (GMSK) for the same values of $B_{b} T$ is also shown in Table 2 as given by Murota and Hirade [3].

Tables 1 and 2 reveal that using GMSK is more efficient than LMSK under the definition of $99.99 \%$ bandwidth (i.e., $99.99 \%$ of the power inside the bandwidth) and using LMSK is more efficient than GMSK under the definition of $90 \%, 99 \%$, and $99.9 \%$ bandwidth.

Table 1. Occupied RF bandwidth containing a given percentage power for LMSK

\begin{tabular}{|c|c|c|c|c||}
\hline$B_{b} T$ & $\begin{array}{c}\mathbf{9 0} \mathbf{\%} \\
\mathbf{2 W}\end{array}$ & $\begin{array}{c}\mathbf{9 9} \mathbf{\%} \\
\mathbf{2 W}\end{array}$ & $\begin{array}{c}\mathbf{9 9 . 9} \mathbf{9} \\
\mathbf{2 W}\end{array}$ & $\begin{array}{c}\mathbf{9 9 . 9 9} \mathbf{2 W} \\
\mathbf{2 W}\end{array}$ \\
\hline 0.2 & $0.47 R_{b}$ & $0.77 R_{b}$ & $0.99 R_{b}$ & $1.31_{R_{b}}$ \\
\hline 0.25 & $0.52 R_{b}$ & $0.83 R_{b}$ & $1.05 R_{b}$ & $1.7 R_{b}$ \\
\hline 0.5 & $0.65 R_{b}$ & $0.95 R_{b}$ & $1.34_{R_{b}}$ & $2.4 R_{b}$ \\
\hline $\operatorname{MSK}\left(B_{b} T=\infty\right)$ & $0.72 R_{b}$ & $1.2 R_{b}$ & $2.76 R_{b}$ & $6.0 R_{b}$ \\
\hline
\end{tabular}

Table 2. Occupied RF bandwidth containing a given percentage power for GMSK [3].

\begin{tabular}{|c|c|c|c|c|}
\hline$B_{b} T$ & $\begin{array}{c}90 \% \\
2 \mathrm{~W}\end{array}$ & $\begin{array}{c}99 \% \\
2 W\end{array}$ & $\begin{array}{c}99.9 \% \\
2 W\end{array}$ & $\begin{array}{c}99.99 \% \\
2 W\end{array}$ \\
\hline 0.2 & $0.52 R_{b}$ & $0.79_{R_{b}}$ & $0.99_{R_{b}}$ & $1.22 R_{b}$ \\
\hline 0.25 & $0.57 R_{b}$ & $0.86_{R_{b}}$ & $1.09_{R_{b}}$ & $1.37 R_{b}$ \\
\hline 0.5 & $0.69_{R_{b}}$ & $1.04_{R_{b}}$ & $1.33 R_{b}$ & $2.08_{R_{b}}$ \\
\hline $\operatorname{MSK}\left(B_{b} T=\infty\right)$ & $0.72 R_{b}$ & $1.2 R_{b}$ & $2.76 R_{b}$ & $6.0 R_{b}$ \\
\hline
\end{tabular}

\section{BIT-ERROR RATE PERFORMANCE OF LMSK}

Let us now consider the bit-error-rate (BER) performance of LMSK. In practice, good approximation for evaluating the SNR at the decision timing is based on the worstcase signal pattern which yields the minimum eye opening. In the case of GMSK, one of the worst signal patterns is .... M, M, S, S ..., where $M$ and $S$ denote the 
phase changes corresponding to those caused by mark and space frequencies (i.e., transmitting long sequence of +1 or -1 ), respectively, in the MSK case [4].

Thus, for LMSK following [4] to evaluate the equivalent SNR degradation from MSK where the Gaussian LPF is replaced by Laplacian LPF. The method of computing SNR degradation is not shown here but just the theoretical results are plotted in Fig. 6 versus the normalized 3-dB bandwidth $B_{b} T$ of Laplacian LPF regardless of $P_{e}$. This theoretical SNR degradation is presented here just for comparison with SNR degradation of GMSK given by Ishizuka and Yashuda [4] which is plotted in the same figure, and also to compare them with SNR degradation obtained by simulation. Fig. 6 reveals that the SNR degradation of LMSK from MSK is lower than that of GMSK which results in less error rate in using Laplacian premodulation filter than Gaussian premodulation filter with the same normalized 3-dB bandwidth $B_{b} T$.

The simulation is carried out for Laplacian filtered MSK and also for Gaussian filtered MSK through a linear channel, for the same memory factor. The SNR degradation of LMSK and GMSK from MSK are computed from simulation results at BER of $10^{-4}$ $\left(P_{e}=10^{-4}\right)$, and plotted in Fig.7; while the bit-error-rate curves versus SNR for LMSK are shown in Fig. 8 for various values of $B_{b} T$. From Fig. 7 we can see that the SNR degradation of LMSK from MSK is lower than that of GMSK which is the same result obtained from the theoretical SNR degradation obtained earlier in Fig.6.

These results reveal that using LMSK is more efficient under the criteria of error rate performance than GMSK (i.e., BER of LMSK is lower than that of GMSK at the same value 3-dB bandwidth $B_{b} T$.

\section{CONCLUSIONS}

The proposed Laplacian filtered-MSK (LMSK) attain good improvement in spectral efficiency over MSK; and in using LMSK we have also good improvement in power efficiency (error rate) over Gaussian filtered-MSK. The improvement achieved by the proposed filtering has been evaluated using simulation results.

\section{REFERENCES}

[1] Rowe, H. E. and Prabhu, V. K., "Power Spectrum of a Digital, Frequency Modulation Signal," Bell System Tech. Journal, Vol.54, No.6, pp.1095-1125, (1975).

[2] De Jager, F. and Dekker, C. B., "Tamed frequency modulation-A novel method to achieve spectrum economy in digital transmission," IEEE Trans. Communication, Vol.COM-26, No.5, pp.534-542, (1978).

[3] Murota, K. and Hirade, K., "GMSK modulation for digital mobile telephony," IEEE Trans. Communication, Vol.COM-29, No.7, pp. 1044-1050, (1981).

[4] Ishizuka, M. and Yasuda, Y., "Improved Coherent Detection of GMSK." IEEE Trans. Communication, Vol.COM-32. No.3, pp.308-311, (1984).

[5] Asano, D. and Pasupathy, S., "Optimization of Coded GMSK Systems," IEEE Trans. Inf. Theory., vol.48, no.10, pp.2768--2773, (2002).

[6] Ziemer, R. E. and Ryan, C. R., "Minimum-Shift Keyed Modem Implementations 
for High Data Rates," IEEE Communication Mag., Vol.21, pp.28-37, (1983).

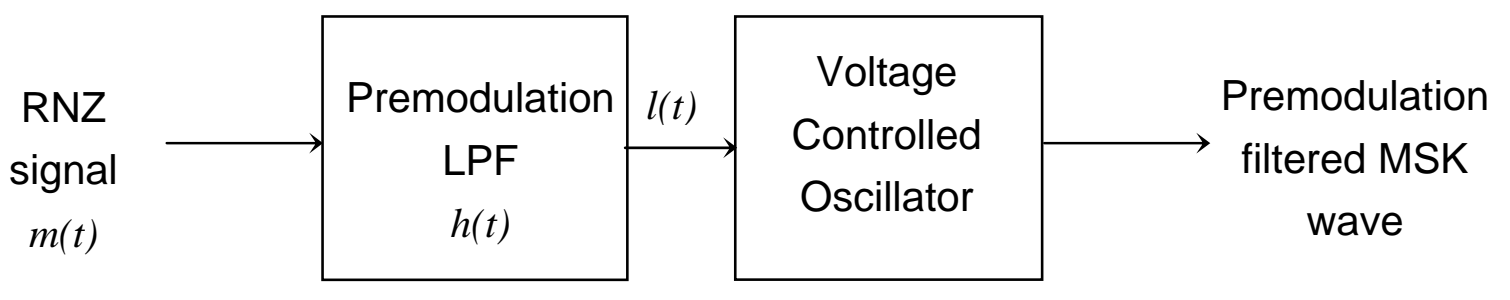

Fig.1. Premodulation filtered MSK modulator.

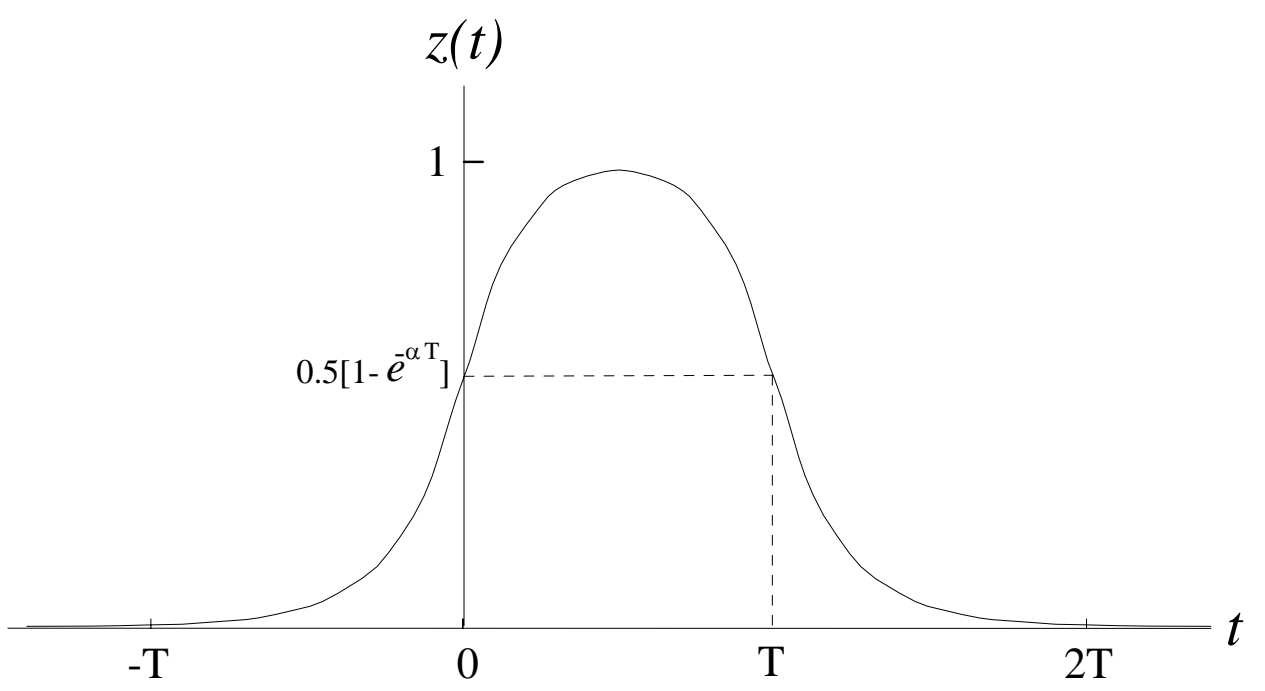

Fig.2. The modulating signal $z(t)$.

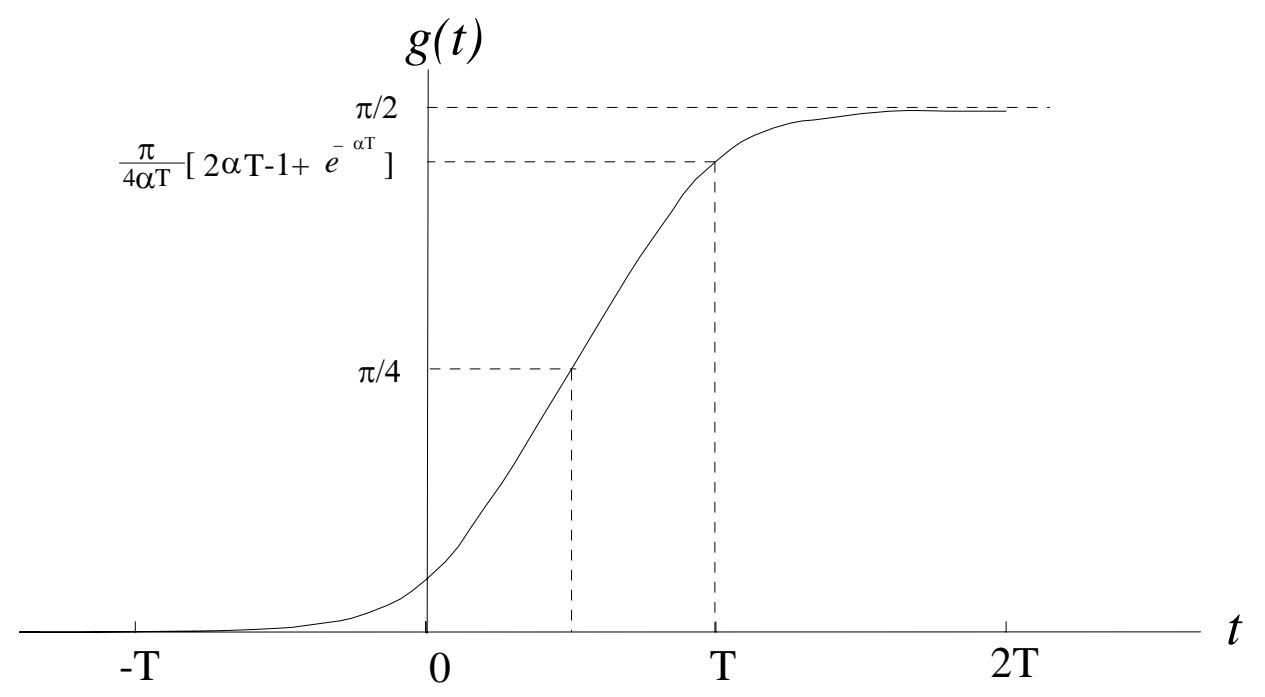

Fig.3. The phase deviation $\mathrm{g}(\mathrm{t})$. 


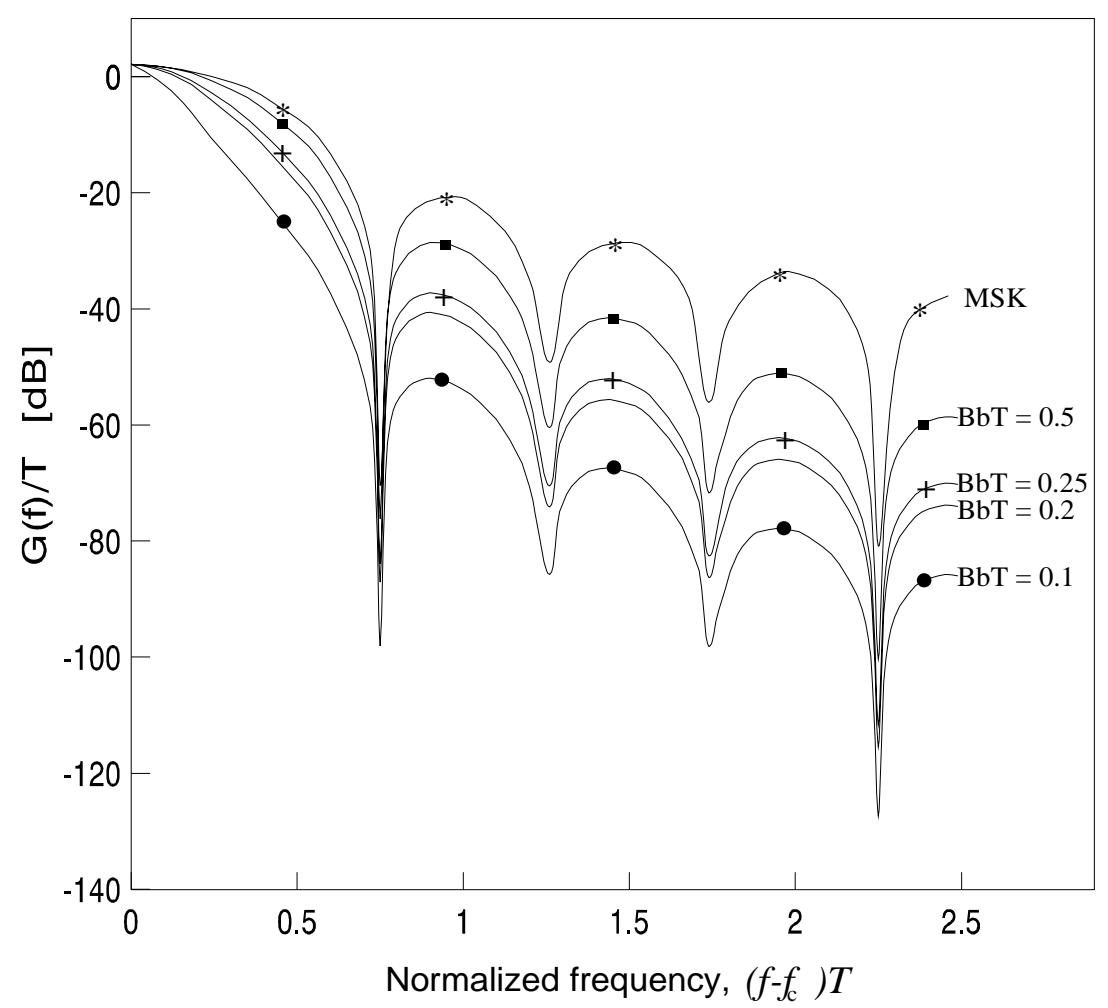

Fig.4. Power spectra of LMSK for various values of $B_{b} T$.

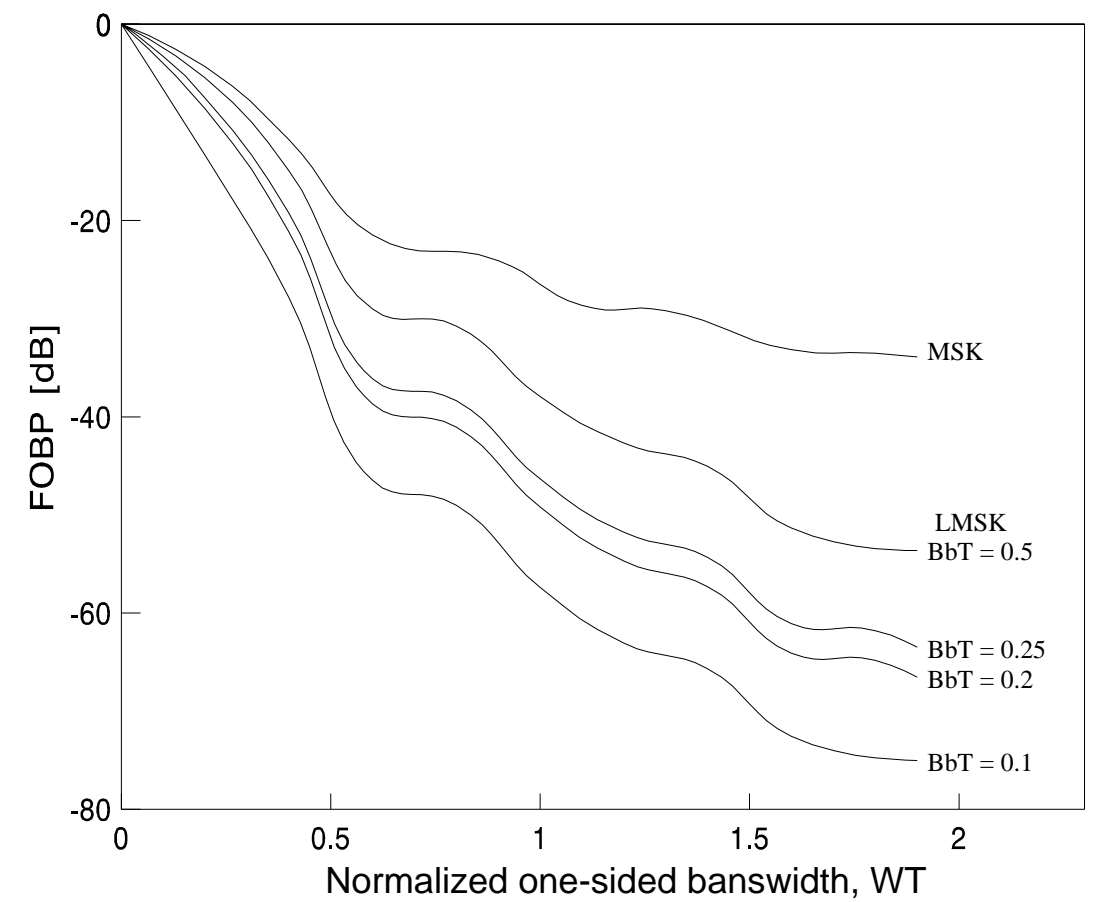

Fig.5. Fractional out-of-band power of LMSK for various values of $B_{b} T$. 


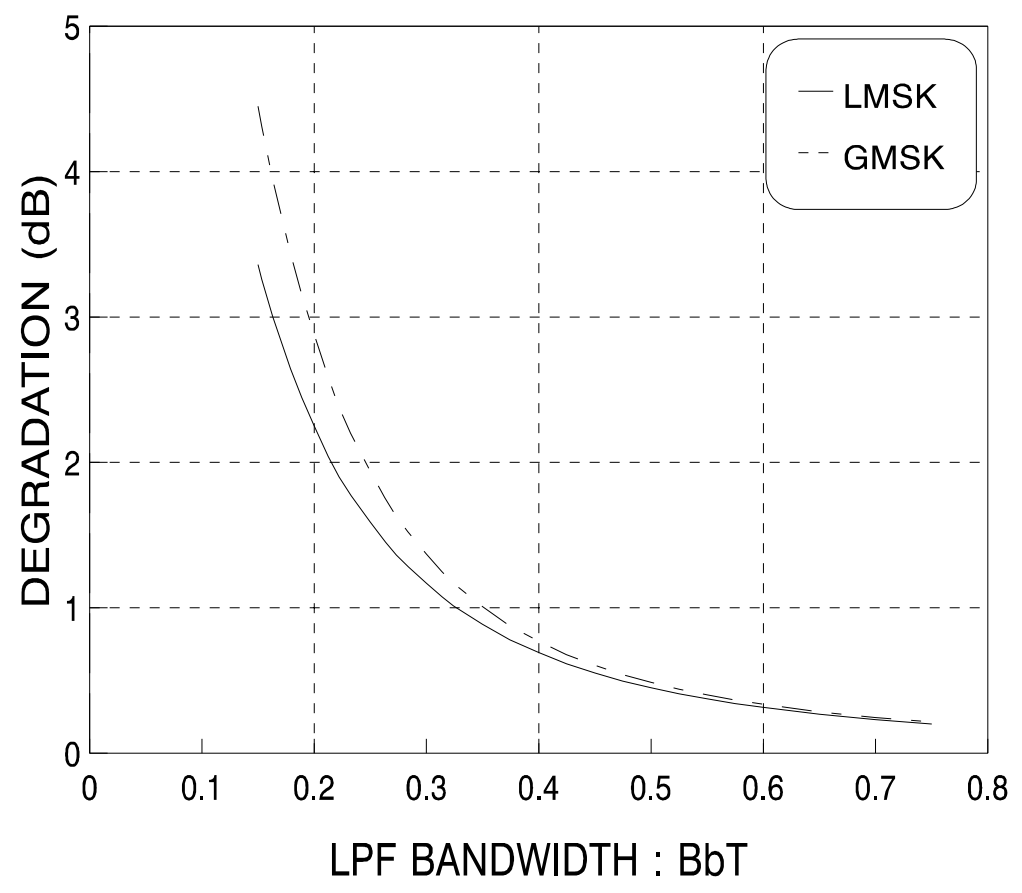

Fig.6. Theoretical SNR degradation of GMSK [6] and LMSK.

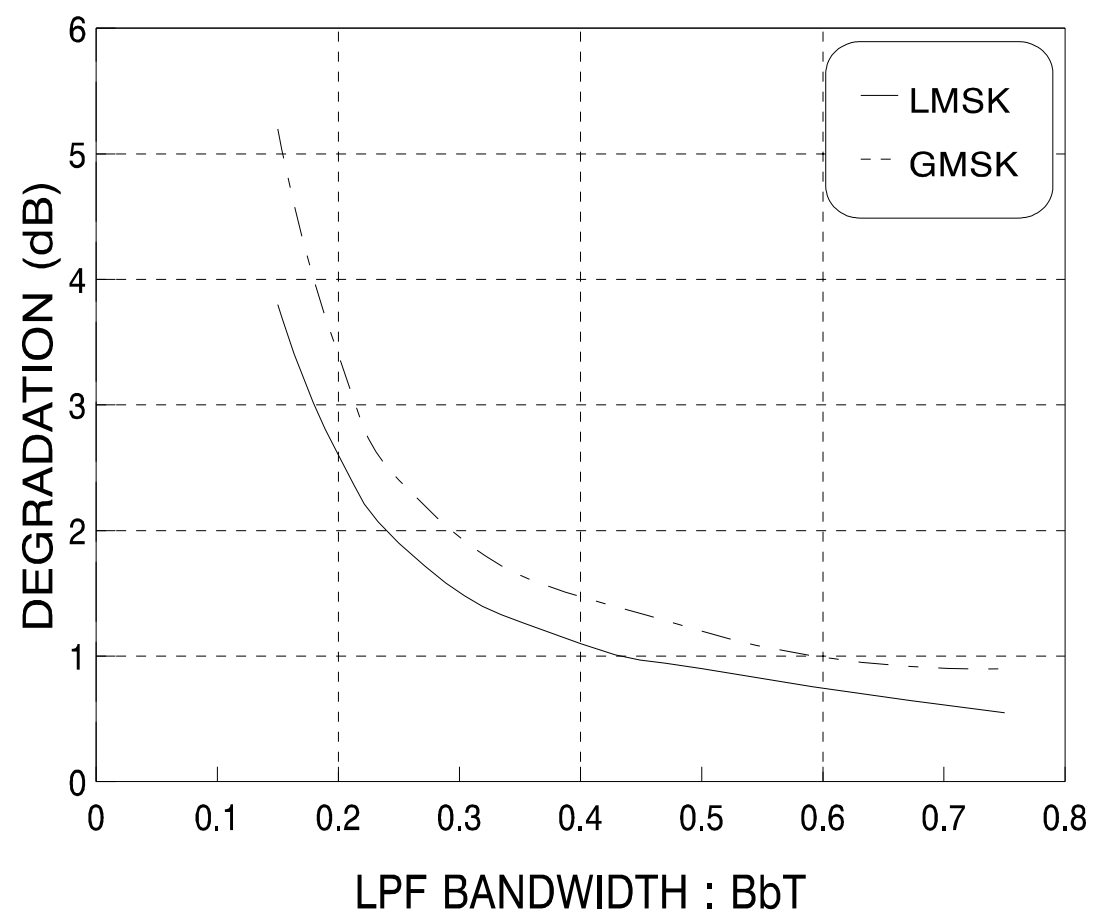

Fig.7. SNR degradation of GMSK and LMSK at $P_{e}=10^{-4}$ (simulation results). 


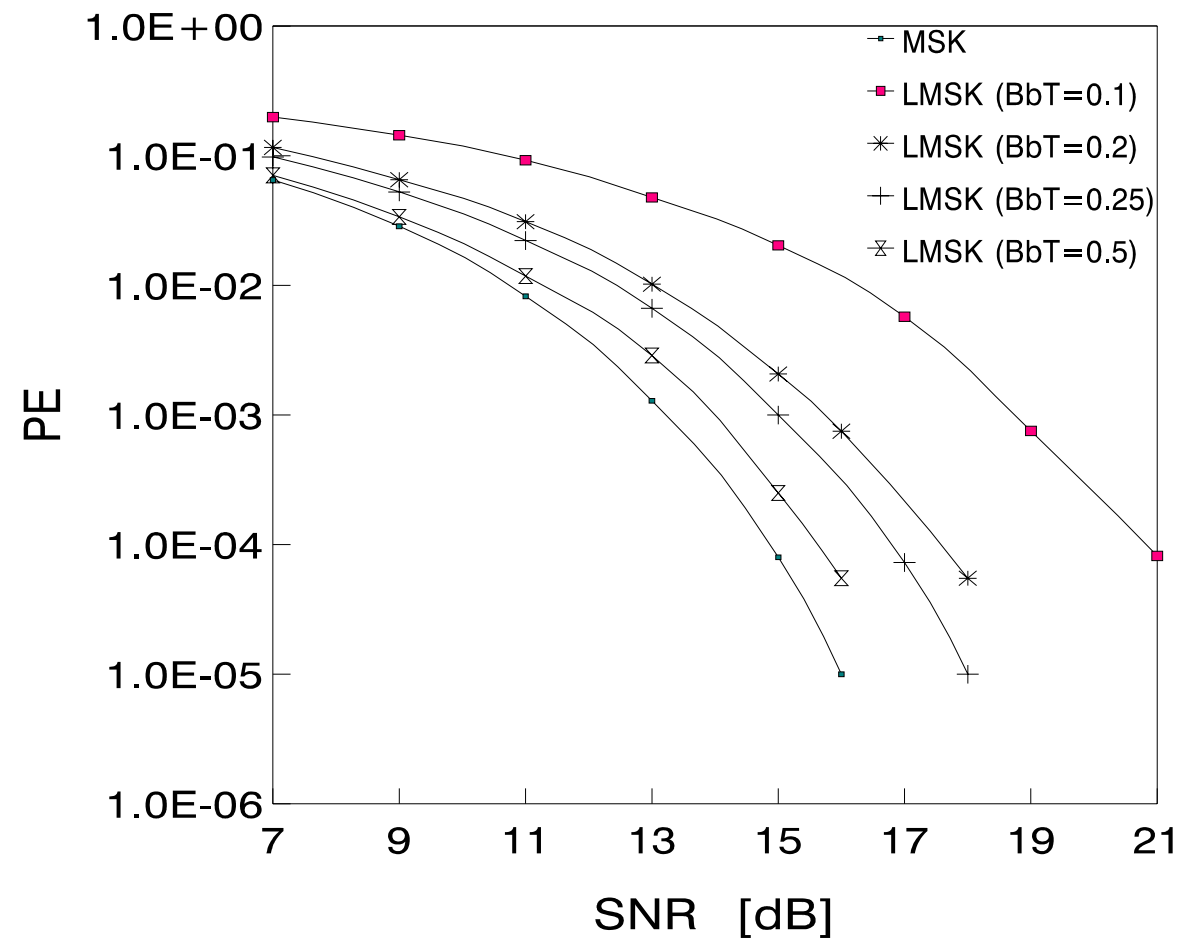

Fig.8. Bit-error-rate for LMSK in a discrete linear channel for various values of 3-dB bandwidth $B_{b} T$. 